\title{
Early palliative interventions for improving outcomes in people with a primary malignant brain tumour and their carers (Protocol)
}

Byrne A, Sivell S, Moraes FY, Bulbeck H, Torrens-Burton A, Bernstein M, Nelson A, Fielding H

Byrne A, Sivell S, Moraes FY, Bulbeck H, Torrens-Burton A, Bernstein M, Nelson A, Fielding H.

Early palliative interventions for improving outcomes in people with a primary malignant brain tumour and their carers.

Cochrane Database of Systematic Reviews 2019, Issue 9. Art. No.: CD013440.

DOI: 10.1002/14651858.CD013440.

www.cochranelibrary.com

Early palliative interventions for improving outcomes in people with a primary malignant brain tumour and their 
TABLE OF CONTENTS

HEADER 1

ABSTRACT

BACKGROUND

OBJECTIVES

METHODS

ACKNOWLEDGEMENTS

REFERENCES

APPENDICES

CONTRIBUTIONS OF AUTHORS

DECLARATIONS OF INTEREST

SOURCES OF SUPPORT 
[Intervention Protocol]

\section{Early palliative interventions for improving outcomes in people with a primary malignant brain tumour and their carers}

Anthony Byrne1,2, Stephanie Sivell2, Fabio Ynoe Moraes ${ }^{3}$, Helen Bulbeck ${ }^{4}$, Anna Torrens-Burton², Mark Bernstein ${ }^{5}$, Annmarie Nelson², Helen Fielding6

${ }^{1}$ Cardiff and Vale University Health Board, Llandough Hospital, Penarth, UK. 2Marie Curie Palliative Care Research Centre (MCPCRC), Division of Population Medicine, School of Medicine, Cardiff University, Cardiff, UK. ${ }^{3}$ Department of Oncology, Division of Radiation Oncology, Kingston Health Sciences Centre, Kingston, Canada. ${ }^{4}$ Director of Services, brainstrust, Cowes, UK. ${ }^{5}$ Faculty of Medicine,

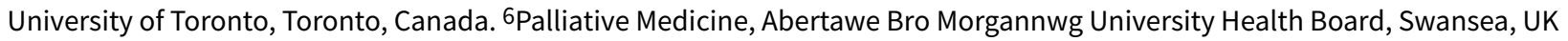

Contact address: Anthony Byrne, Cardiff and Vale University Health Board, Llandough Hospital, Penlan Road, Penarth, Vale of Glamorgan, CF64 2XX, UK. Anthony.Byrne2@wales.nhs.uk.

Editorial group: Cochrane Gynaecological, Neuro-oncology and Orphan Cancer Group

Publication status and date: New, published in Issue 9, 2019.

Citation: Byrne A, Sivell S, Moraes FY, Bulbeck H, Torrens-Burton A, Bernstein M, Nelson A, Fielding H. Early palliative interventions for improving outcomes in people with a primary malignant brain tumour and their carers. Cochrane Database of Systematic Reviews 2019, Issue 9. Art. No.: CD013440. DOI: 10.1002/14651858.CD013440.

Copyright @ 2019 The Cochrane Collaboration. Published by John Wiley \& Sons, Ltd.

\section{A B S T R A C T}

This is a protocol for a Cochrane Review (Intervention). The objectives are as follows:

To assess the evidence base for early palliative care interventions, including referral to specialist palliative care services for improving outcomes in people diagnosed with a primary brain tumour and their carers. 


\section{B A C K G R O U N D}

\section{Description of the condition}

Primary brain tumours account for an estimated $2 \%$ of malignancies worldwide (Ferlay 2015). Approximately 5000 people are diagnosed with a primary malignant brain tumour each year in the UK (Cancer Research UK 2015). Gliomas are the most common type of primary brain tumour, accounting for up to $80 \%$ of malignant brain tumours overall (Goodenberger 2012). Gliomas are graded from 1 to 4 according to the World Health Organization (WHO) classification (Louis 2016). Grades 1 and 2 are low-grade slow-growing tumours. Grades 3 and 4 are high-grade fast-growing tumours. Highgrade primary malignant glioma occurs most commonly between the fifth and seventh decades (Stupp 2010). It is a particularly aggressive disease, with a median survival time of 12 to 15 months (Stupp 2009). The five-year survival rate is 10\% (Tran 2010).

The symptom burden for people diagnosed with a high-grade glioma is substantial. There can be profound effects on physical, neurocognitive, and social functioning from an early stage in the illness (Faithfull 2005; Long 2016; Moore 2013). Neurological deficits can include motor weakness, epilepsy, and dysphasia. This population of patients also frequently experience personality changes, a decrease in mental capacity, and mood disturbance. These effects can be exacerbated by aggressive treatment regimens (Aziz 2003; Long 2016).

The disease trajectory is unpredictable. It is often characterised by periods of sudden acute deterioration followed by a period where the clinical condition appears to plateau (Philip 2015). This makes prognostication difficult. Patients become increasingly dependent and isolated, which combined with the symptom burden can result in a reduction in perceived quality of life.

Informal care providers of patients with high-grade glioma are reported to experience significant burden and distress (Jacobs 2014; Wasner 2013). Collins 2014 reported significant needs in relation to the challenge of caring, the lack of support available to carers, and the suffering of caregivers (Collins 2014). The neurocognitive effects of the disease coupled with the increased dependency and social isolation can result in changes to relationships with family members/care providers, which are not so commonly observed in the context of other malignancies (Ford 2012; Lipsman 2007).

Palliative care seeks to improve the quality of life of patients and families facing a life-limiting illness through the identification, assessment, and treatment of physical, psychosocial, and spiritual needs (NCHSPCS 2002). In addition, it provides assistance with decision making and supports caregiver burden and well-being. Specialist palliative care has specific areas of expertise that can address complex symptoms, psychosocial, end-of-life, and bereavement issues for patients with a life-limiting illness (NCHSPCS 2002). Specialist palliative care teams are multi-disciplinary in nature and specifically trained to deal with multidimensional problems, such as those encountered in high-grade glioma.

Demonstrating the benefit of specialist palliative care in improving patient and carer-reported outcomes is an ongoing focus of research. A systematic review by Higginson 2010 concluded that home, hospital, and inpatient specialist palliative care teams significantly improved patient outcomes in the domains of pain and symptom control, anxiety, and reduced hospital admissions for cancer patients. There is a need to better understand the effects of different models of palliative care. Initiatives, such as the European-funded collaborative PRISMA, have focused on establishing standardised outcome measures for palliative care to improve the quality of research in this field (Harding 2010).

Previous studies have highlighted the lack of existing evidence surrounding the services needed to support this patient cohort and their care providers (Ford 2012; Moore 2013). The timing of such interventions has also not been well-defined. The National Institute for Health and Clinical Excellence (NICE) recommends that patients with a primary brain tumour could benefit from specialist palliative and supportive care early in the process, at diagnosis if possible, with continued integration of services throughout the course of the patient's illness (NICE 2018). However, the provision of palliative and supportive care for this patient population has historically been ill-defined and ad hoc (Faithfull 2005; Moore 2013), and the nature of the interventions that are most beneficial has not been confirmed.

\section{Description of the intervention}

The emphasis of palliative care is on dealing with the whole person: identifying and managing physical, psychological, and spiritual symptoms that profoundly affect quality of life. It also focuses on assistance with decision making, including advance care planning and addressing issues of relevance to significant others in terms of caregiver burden and well-being (Radbruch 2009; Rietjens 2017; WHO 2018).

Palliative care interventions may be initiated individually by the oncologist, neurosurgeon, or primary care team; by members of the wider supportive care team in a coordinated care approach; or be provided by specialist palliative care teams as part of an integrated model. A specialist palliative care service is defined as one delivered by a multi-professional team, usually comprising of doctors, nurses, and psychosocial workers with higher training in palliative care provision, possibly commissioned to provide palliative care at a specialist level.

The concept of 'early' palliative care has been introduced more recently to differentiate palliative interventions delivered earlier in the disease trajectory from those in the terminal phase or last days of life. There is, however, no universally accepted definition, with significant heterogeneity in description of what constitutes 'early' in reported studies in cancer and in other serious illnesses. This has ranged from definitions based on time since diagnosis or recurrence (Bakitas 2015), likely prognosis (Zimmermann 2014), in tandem with oncological review (Temel 2010), or based on symptom burden (Groenvold 2017). The American Society of Clinical Oncology practice guidelines suggest a definition of as early as "within 8 weeks of diagnosis" (Ferrell 2016).

This Cochrane Review will focus on palliative care interventions either in the form of a specialist palliative care service or interventions undertaken by other healthcare professionals with the specific intent of palliation. The review will include interventions delivered to both the person with the brain tumour and the carer or either alone. It will include interventions delivered in both community and secondary care settings. The timing, nature, and duration of the intervention must be clearly stipulated. Studies included in this review will include an explicit intent to provide 'early palliative care' or provide a clear study definition of 'early' in relation to time 
since diagnosis or provision during ongoing active anticancer intervention.

\section{How the intervention might work}

People diagnosed with a primary brain tumour often experience significant disability early on in their illness. A wide range of physical symptoms have been reported in existing literature including fatigue, pain, seizures, and cognitive impairment (Armstrong 2016; Faithfull 2005; Ford 2012). Depression and anxiety are also common. A longitudinal study of 600 patients by Batchelor 2006 identified that $15 \%$ of people with a malignant glioma had depression in the early postoperative period and a further $93 \%$ described depressive symptoms. A systematic review by Ford 2012 reported prevalence rates of depression and anxiety in up to $48 \%$ of people diagnosed with a primary malignant brain tumour.

A recent systematic review by Moore 2013 reviewed qualitative literature looking at the palliative and supportive care needs of people with high-grade glioma and their care providers. Key themes identified included: the need for consistent, well-delivered information around disease sequelae, treatment, and resources available; health service needs including key professionals to coordinate care; the need for psychological and social support and clear avenues of communication with treating professionals. Vierhout 2017 conducted a qualitative study that explored the views of people diagnosed with a brain tumour on palliative care. A key theme identified was that people were keen to be aware of palliative care options early in their illness.

In the wider literature there is increasing evidence that palliative care interventions are associated with improved patient outcomes in both malignant and non-malignant life-limiting conditions (Bakitas 2015; Harding 2010; Higginson 2010; Kristjanson 2006; Temel 2010; Temel 2017; Zimmermann 2014), although not all studies demonstrate benefit (Davis 2015; Groenvold 2017). The systematic review by Davis 2015 highlighted significant heterogeneity in patient populations, intervention types, settings, and outcome measurements making comparisons difficult. Nonetheless, a recent random-effects meta-analysis of palliative care interventions on patient and carer outcomes by Kavalieratos 2016 included 43 studies across a range of conditions and demonstrated improvements in patient quality of life and symptom burden. A more recent Cochrane Review compared early palliative care interventions with more standard treatment care in advanced cancer (but not specifically brain cancer) and again observed a significant improvement in quality of life and symptom burden in patients receiving care shortly after diagnosis compared to closer to end of life (Haun 2017). An unexpected finding in some studies has been the presence of a survival advantage in those receiving early palliative care intervention (Bakitas 2015; Temel 2010). Although this has not been replicated in the Kavalieratos 2016 or Haun 2017 reviews, the importance of assessing for survival, and exploration of potential underlying reasons, has been highlighted.

\section{Why it is important to do this review}

People diagnosed with a primary brain tumour experience a high symptom burden, uncertain prognosis, and unpredictable disease trajectory. This is often characterised by rapid physical and neurocognitive decline that can place significant burden on care providers. Specialist palliative care services are well-placed to be able to support the complex needs of this patient population. How- ever there are currently no systematic reviews that have looked specifically at the evidence base for early referral to specialist palliative care services or other designated early palliative care interventions for improving quality of life, carer outcome, and overall survival in patients diagnosed with a primary brain tumour.

Previous studies that have looked at the supportive and palliative care needs of patients diagnosed with a high-grade glioma have consistently concluded that the quality of evidence remains limited (Catt 2008; Collins 2014; Lin 2012; Moore 2013). In particular, there has been a lack of studies conducted in a palliative care setting. There is low-quality evidence to support multidisciplinary rehabilitation in reducing short- and long-term disability in patients with brain tumours compared to best supportive care (Khan 2015). Collins 2014 concluded that carers of patients with primary malignant glioma have distinct supportive and palliative care needs which differ from those of other cancer trajectories, although the existing literature has yet to define how these needs might be best addressed, by whom and at what point in a patient's care (McConigley 2012). Defining the nature of effective interventions in this context will help develop a more collaborative, needs-based model of care.

The importance of this topic is recognised at a national level. The National Institute for Health and Clinical Excellence (NICE) recommends that people with brain tumours could benefit from specialist palliative and supportive care early in the process, at diagnosis if possible with continued integration throughout the course of the person's illness (NICE 2006). NICE also recommend the co-operation of neuro-oncology and specialist palliative care to "ensure an appropriate balance between treatment and palliative care" (NICE 2006 p.121). Through doing so, NICE anticipates that not only will communication be improved, but service provision will be more responsive to patients' needs with more timely transfer of patients from services and treatments, patients, and their families/caregivers will be more satisfied and patients may be able to stay in their preferred place of care through improved continuity of care. The recent James Lind Alliance Neuro-Oncology Priority Setting Partnership Report gives context to the priority of research in this area (MacDonald 2015).

Ultimately, understanding the role early referral to specialist palliative care services or effectiveness, or both of other palliative care interventions has on the parameters outlined would help guide improvement to service provision and the development of an evidence-based model of supportive and palliative care for this patient population.

\section{O B JECT IVES}

To assess the evidence base for early palliative care interventions, including referral to specialist palliative care services for improving outcomes in people diagnosed with a primary brain tumour and their carers.

\section{METHODS}

\section{Criteria for considering studies for this review Types of studies}

We will include randomised controlled trials and non-randomised intervention studies. We will also include qualitative studies and mixed-methods studies where both qualitative and quantitative 
data is included. Non-randomised trials are susceptible to greater risk of bias so to ensure an acceptable quality of included non-randomised studies, we will scrutinise risk of bias using the ROBINS-I tool (Sterne 2016), specifically designed for this purpose.

\section{Types of participants}

\section{Inclusion criteria}

- Adults (aged 18 years and older) who have a confirmed radiological or histological diagnosis, or both, of a primary malignant brain tumour.

- Informal adult carers of people with a confirmed diagnosis of a primary malignant brain tumour. This will usually be at an individual level but we will also include family level.

\section{Exclusion criteria}

- Participants who have a diagnosis of a benign brain tumour.

- Participants who have metastatic disease from an extra cranial primary.

\section{Types of interventions}

We will include studies where there is explicit intent to provide 'early palliative care' or where there is a clear study definition of 'early' in relation to time since diagnosis or ongoing active anticancer intervention. We will include studies reporting specialist and nonspecialist palliative care intervention defined as any intervention by a healthcare professional that addresses palliation in any or all of the following areas:

- Symptom control.

- Physical function.

- Cognitive function.

- Psychological support.

- Information giving.

- Advance or future care planning.

A specialist palliative care service is defined as one delivered by member(s) of a multi-professional team with higher training in palliative care provision, or commissioned to provide palliative care at a specialist level, or both.

We will include interventions delivered in community and secondary care settings, and interventions delivered to both participant and carer or either alone. We will only include interventions where the specific components of an intervention are described and the timing (commencement) and duration of the intervention are clearly stipulated.

The comparators of interest will be usual care, including as part of a waiting list control. Usual care is defined as that normally provided by the neuro-oncology team. It might include provision of generalist or specialist palliative care support but not intentionally activated for all people at the time of diagnosis or initiation of anticancer treatment.

\section{Types of outcome measures}

We will include trials looking at early referral to specialist palliative care services, or early targeted palliative interventions by other healthcare professionals for improving quality of life, symptom control, carer outcomes, or overall disease survival as a primary or secondary outcome measure.

\section{Primary outcomes}

The primary outcomes will be quality of life, symptom control, psychological outcomes, and overall survival. We will report outcomes separately for participants and, where appropriate, carers in 'Summary of findings' tables using GRADEpro software (GRADEpro GDT 2014). Further details on the outcome measures that we will accept, where reported by included studies, are shown below.

\section{Participants}

- Survival from time of enrolment, to include one year and overall survival.

- Quality of life using validated Quality of Life tools e.g. FACTG (Cella 1993) and FACT- Br (Weitzner 1995), EORTC QLQ C30 and BCM 20 (Osoba 1996), McGill Quality of Life Questionnaire (Cohen 1995), 36-Item Short Form Health Survey (SF-36) (Ware 1992), 46-item Functional Assessment of Chronic Illness Therapy- Palliative Care (FACIT-Pal) (Lyons 2009); qualitative analysis of participant experience using validated and clearly described methodologies.

- Symptom control using validated symptom assessment tools e.g. Edmonton Symptom Assessment Scale (ESAS) (Bruera 1991); Palliative Outcomes Scale (POS) (Hearn 1999); Quality of Life at the End of Life (QUAL-E) (Steinhauser 2004); Symptom Experience Scale (SES) (Samarel 1996); physical and cognitive function using validated assessment tools.

- Psychological outcomes including anxiety and depression using validated assessment tools e.g. Beck Depression Inventory (BDI) (Beck 1961), Hamilton Rating Scale for Depression (HAM-D) (Hamilton 1960), Hospital Anxiety and Depression Scale (HADS) (Zigmond 1983).

\section{Informal carer(s)}

- Psychological outcomes including anxiety and depression using validated assessment tools (as mentioned above; carer burden using validated assessment tools e.g. Caregiver Strain Index (CSI) (Robinson 1983), Supportive Care Needs Survey for Partners \& Caregivers (SCNS-P\&C) (Girgis 2011), the Carer Experience scale (CES) (Al-Janabi 2008), Quality of Life During Serious Illness-Family Carers (QOLLTI-F) (Cohen 2006), Zarit Burden Inventory (Seng 2010), and FAMCARE (Kristjanson 1993)).

\section{Secondary outcomes}

The secondary outcomes will be: care coordination and information giving by participants and carers, receipt of planned anticancer treatment for participants, bereavement outcomes for informal carers, carer experience, and resource use and costs. Further details on the outcome measures that we will accept, where reported by included studies, are shown below.

\section{Participants}

- Care coordination and information giving based on qualitative assessment of participant feedback, or objective measures of satisfaction, or both.

- Receipt of planned anticancer treatment: completion of initial neuro-oncology multidisciplinary team (MDT) treatment. 
- Resource use to include hospital and hospice utilisation measured in length of inpatient stay in days, number of outpatient appointments.

\section{Informal carer(s)}

- Care coordination and information giving based on qualitative assessment or carer feedback or objective measures of satisfaction, or both.

- Qualitative analysis of carer experience using clearly described and validated methodologies.

- Bereavement outcomes using validated measures.

- Resource use and costs. To include opportune costs of loss of income.

Important information on participants and carer experience of interventions may be published as part of a controlled trial or separately.

We will report outcomes separately for participants and carers in 'Summary of findings' tables (Appendix 1; Appendix 2).

\section{Search methods for identification of studies}

\section{Electronic searches}

We will identify relevant studies by conducting searches of electronic databases, and will include the Cochrane Central Register of Controlled Trials (CENTRAL: latest issue), MEDLINE via Ovid (1946 to present), Embase via Ovid (1980 to present), Cumulative Index to Nursing and Allied Health Literature (CINAHL from 1982 to present), Web of Science, and Psychlnfo. We will conduct searches to incorporate both qualitative and quantitative search terms.

We will search for any currently recruiting trials in ClinicalTrials.gov (http://clinicaltrials.gov/) and in the World Health Organization (WHO) International Clinical Trials Registry Platform (ICTRP) search portal (http://apps.who.int/trialsearch/).

The detailed search strategy for Ovid MEDLINE is shown in Appendix 3. For databases other than MEDLINE we will adapt the search strategy accordingly.

\section{Searching other resources}

We will handsearch the reference lists of any included articles to identify any other relevant studies. Experts in the field will be contacted to suggest relevant unidentified studies (published or unpublished). In addition we will find all included articles using citation tracking via Scopus.

We will handsearch the most relevant journals. In addition, we will source Dissertations and Theses, searching key authors via Web of Science and search SIGLE - System for information on grey literature in Europe.

\section{Data collection and analysis}

\section{Selection of studies}

Two review authors (ATB, AB) will independently screen and shortlist all abstracts and study titles identified by the search strategy to assess eligibility against the inclusion criteria. We will obtain fulltext copies of all papers considered to be potentially eligible for further assessment to determine if the study met the inclusion/exclusion criteria. A second review author will then check identified pa- pers (divided between $\mathrm{AB}, \mathrm{MB}, \mathrm{FM}, \mathrm{HB}, \mathrm{AN}$, or $\mathrm{SS}$ ). We will resolve any disagreement by discussion with a third review author if necessary (from AB, MB, FM, HB, AN, or SS) to reach a consensus. If necessary, we will contact the authors of primary papers for clarification. The review authors will not be masked to the name(s) of the author(s), institution(s), or publication source at any level of the review. We will illustrate the study selection process in a PRISMA diagram.

\section{Data extraction and management}

Two review authors (from ATB, AB, MB, FM, HB, AN, or SS) will independently complete the data extraction using a standardised data collection form and a third review author (from ATB, AB, MB, FM, $\mathrm{HB}, \mathrm{AN}$, or SS) will independently check the data to minimise errors and reduce potential bias. We will initially complete data extraction forms electronically for ease of distribution between review authors. In cases where we use a paper copy, one review author (ATB) will type up the extracted data verbatim. We will pilot the data collection forms against the checklist provided in the Cochrane Handbook for Systematic Reviews of Interventions (Higgins 2011), and all review authors will agree the included items. Extracted data will include but not be limited to participants (including sample size), study design, setting, intervention details, outcomes, results, and information to facilitate the assessment of the risk of bias (i.e. sequence generation, allocation sequence concealment, blinding). For multiple reports of the same study, we will collate data into a single collection form. For any data that is missing and cannot be included in the form, we will attempt to obtain by contacting the primary authors.

For all studies that meet the inclusion criteria, we will summarise in the 'Characteristics of included studies' table provided in Review Manager 5 software (Review Manager 2014). This will include details on design, participants, interventions, and outcomes. For nonrandomised studies, we will provide appropriate additional study features in line with the Cochrane Handbook for Systematic Reviews of Interventions (Reeves 2011), such as any comparisons included, how groups were created, and which aspects of the study were prospective.

\section{Assessment of risk of bias in included studies}

Two review authors (from ATB, AB, MB, FM, HB, AN, or SS) will independently assess the risk of bias using the criteria and guidelines from the 'Risk of bias' tool described in the Cochrane Handbook for Systematic Reviews of Interventions (Shuster 2011), which a third review author (ATB, AB, MB, FM, HB, AN, or SS) will independently check. We will report the following seven domains: allocation concealment (checking if allocation methods could be foreseen); sequence generation (checking method of generating allocation sequence); blinding (participants and personnel i.e. methods to blind participants' knowledge of which intervention given); blinding (outcome assessment i.e. blinding assessors from which intervention given to the participant); incomplete outcome data (checking differences between intervention and control groups); selective outcome reporting (checking how outcomes are reported); and 'other bias' (including recruitment bias, stopping early for benefit, carry-over effects in cross-over studies, and non-validated outcome measures).

We will give each domain a judgement of risk of 'low risk', 'high risk', or 'unclear risk' if insufficient details are provided in the study. This will be accompanied with a 'Support for judgement' statement 
summarising how we made risk judgements to ensure transparency of the decisions made. We will consider studies to be of high methodological quality if the risk of bias for all domains is low. We will deem these 'high-quality' studies. We will consider studies to be of low methodological quality if the risk of bias is high or 'unclear' one or more domains. We will deem these 'low-quality' studies. We will resolve any disagreement on the judgement of bias by discussions between the review authors.

For non-randomised studies, we will use the Cochrane Handbook for Systematic Reviews of Interventions for guidance for the types of biases these types of studies are susceptible to which are similar to randomised trials i.e. blinding (outcomes) and for biases that cannot be compared with those randomised studies i.e. allocation methods. We will assess risk of bias using the ROBINS-I tool (Sterne 2016), designed specifically for a non-randomised design.

\section{Measures of treatment effect}

We will enter and analyse all of the quantitative data in Review Manager 5 (Review Manager 2014). Where possible we will calculate risk ratios (RRs) with 95\% confidence intervals (Cls) for dichotomous data, and mean differences or standardised mean differences with 95\% Cls for continuous data where different scales are used across trials. For time-to-event data for survival, we will analyse as death hazard ratios under the assumption that the hazard ratio is consistent across the follow-up period. Where data aggregation is not possible, we will present the results of individual studies in table format and describe the primary findings narratively.

\section{Unit of analysis issues}

We anticipate that the appropriate unit of analysis will be by type, timing, and duration of specialist palliative intervention for improving quality of life, carer outcomes, and survival in people diagnosed with a primary brain tumour. We anticipate a limited number of randomised controlled trials and non-randomised intervention studies.

\section{Dealing with missing data}

Where data is missing, we will contact the primary study authors in an attempt to obtain this data. Where data is missing and cannot be derived by contacting the primary authors, we will undertake a complete case analysis. If the data remains missing, we will report the study but not include it in the final analysis.

\section{Assessment of heterogeneity}

We will follow the statistical analysis method as described in the Cochrane Handbook for Systematic Reviews of Interventions (Shuster 2011). We expect heterogeneity due to differences in participant populations, types and timing of interventions, and differences in outcome scales used. We will assess for the presence of variation in effects observed across studies using a $\mathrm{Chi}^{2}$ test. To quantify the degree of heterogeneity we will employ the $1^{2}$ statistic, which reflects the percentage of variability in effect estimates that is due to heterogeneity rather than to chance (Deeks 2011). We will consider a $0 \%$ to $40 \%$ threshold to be a low level of heterogeneity, $30 \%$ to $60 \%$ to be a moderate level of heterogeneity, and $50 \%$ to $90 \%$ to be a substantial level of heterogeneity as suggested by the Cochrane Handbook for Systematic Reviews of Interventions (Deeks 2011). We will also describe, where possible, the potential sources of heterogeneity rather than simply quantify its existence. Non-randomised studies would be expected to be more heterogeneous compared to randomised trials and the most effective method of observing variation is through the visual inspection of the forest plots.

\section{Assessment of reporting biases}

We will aim to minimise publication bias by sourcing unpublished data where possible. If we identify an individual meta-analysis containing at least 10 studies, we will assess publication bias using funnel plots and by Egger's test (Egger 1998).

\section{Data synthesis}

We will conduct preliminary synthesis by entering all data into Review Manager 5 (Review Manager 2014). The included studies will be summarised using the 'Characteristics of included studies' table provided by the Review Manager 5 software. Where there is substantial and unexplained heterogeneity $(P<0.10)$, we will consider pooling data using the random-effects model.

Where studies compare more than one intervention or a combination of interventions, we will analyse each comparison separately. If possible we will calculate a weighted treatment effect using Review Manager 5 software. We will express the results as risk ratios with $95 \% \mathrm{Cls}$ for dichotomous outcomes and mean differences and 95\% Cls for continuous outcomes.

We will describe the qualitative data alongside the quantitative data and where appropriate correlate findings for example in terms of possible domains of impact, and explorations of heterogeneity.

\section{Summary of findings for assessing the certainty of the evidence}

We will present the overall certainty of the evidence for each outcome according to the GRADE approach, which takes into account issues not only related to internal validity (risk of bias, inconsistency, imprecision, publication bias) but also to external validity, such as directness of results (Langendam 2013; Schünemann 2011). We will use the GRADE approach to assess the certainty of the evidence related to each of the key outcome measures listed in Chapter 12.2 of the Cochrane Handbook for Systematic Reviews of Interventions (Shuster 2011). We will create 'Summary of findings' tables using GRADEpro GDT software (GRADEpro GDT 2014; see Appendix 1 and Appendix 2). We will use the GRADE checklist and GRADE Working Group certainty of evidence definitions (Meader 2014). We will downgrade the evidence from 'high' certainty by one level for serious (or by two for very serious) concerns for each limitation:

- High-certainty: we are very confident that the true effect lies close to that of the estimate of the effect.

- Moderate-certainty: we are moderately confident in the effect estimate. The true effect is likely to be close to the estimate of the effect, but there is a possibility that it is substantially different.

- Low-certainty: our confidence in the effect estimate is limited. The true effect may be substantially different from the estimate of the effect.

- Very low-certainty: we have very little confidence in the effect estimate. The true effect is likely to be substantially different from the estimate of effect

\section{Subgroup analysis and investigation of heterogeneity}

Where sufficient studies and data exist, we will undertake the following subgroup analyses. 
- Tumour type.

- Age group (18 to 70 years and over 70 years).

- Type of intervention (individual vs group), frequency of intervention (less than once a week, once a week, 2 to 3 times a week), and duration of specialist palliative care intervention.

- Time from definitive treatment (surgery, radiotherapy, or chemotherapy) to specialist palliative care intervention.

- Type of treatment received; surgery and adjuvant chemotherapy or radiotherapy, or both; surgery alone; chemotherapy alone; radiotherapy alone; combined chemotherapy and radiotherapy with no surgery.

We will investigate whether the results of subgroups are significantly different by performing the test for subgroup differences available in Review Manager 5 (Review Manager 2014).

\section{Sensitivity analysis}

If we identify heterogeneity across the included studies, we will undertake sensitivity analyses to determine the effect of excluding studies at high risk of bias. In addition we will use sensitivity analyses to explore the effect of the primary aims of the study (early re- ferral to specialist palliative care to improve quality of life, carer outcomes, and overall survival for people diagnosed with a primary brain tumour).

\section{ACKNOWLEDGEMENTS}

We acknowledge Jo Platt (Information Specialist) and Mala Mann (Information Specialist/Systematic Reviewer) for their help with developing the search strategies and Helen Fielding (Consultant in Palliative Medicine) for her work on developing the protocol.

The review authors and Cochrane Gynaecological, Neuro-oncology and Orphan Cancers editorial team are grateful to the following peer reviewers for their time and comments: Andrew Bryant, Johan Koekkoek, Christine Marosi, and Andrea Pace.

This project was supported by the National Institute for Health Research (NIHR), via Cochrane infrastructure funding to the Cochrane Gynaecological, Neuro-oncology and Orphan Cancers Group. The views and opinions expressed therein are those of the review authors and do not necessarily reflect those of the Systematic Reviews Programme, the NIHR, the NHS, or the Department of Health. 


\section{REFERE N CE S}

\section{Additional references}

\section{Al-Janabi 2008}

Al-Janabi H, Coast J, Flynn TN. What do people value when they provide unpaid care? A meta-ethnography with interview follow-up. Social Science \& Medicine 2008;67(1):111-21.

\section{Armstrong 2016}

Armstrong TS, Vera-Bolanos E, Acquaye AA, Gilbert MR, Ladha H, Mendoza T. The symptom burden of primary brain tumors: evidence for a core set of tumor- and treatment-related symptoms. Neuro-Oncology 2016;18(2):252-60. [PUBMED: 26289592]

\section{Aziz 2003}

Aziz NM, Rowland JH. Trends and advances in cancer survivorship research: challenge and opportunity. Seminars in Radiation Oncology 2003;13(3):248-66. [PUBMED: 12903014]

\section{Bakitas 2015}

Bakitas MA, Tosteson TD, Li Z, Lyons KD, Hull JG, Li Z, et al. Early versus delayed initiation of concurrent palliative oncology care: patient outcomes in the ENABLE III randomized controlled trial. Journal of Clinical Oncology 2015;33(13):1438-45.

\section{Batchelor 2006}

Batchelor TT, Byrne TN. Supportive care of brain tumor patients. Hematology/Oncology Clinics of North America 2006;20(6):1337-61. [PUBMED: 17113467]

\section{Beck 1961}

Beck AT, Ward CH, Mendelson M, Mock J, Erbaugh J. An inventory for measuring depression. Archives of General Psychiatry 1961;4(6):561-71.

\section{Bruera 1991}

Bruera E, Kuehn N, Miller MJ, Selmser P, Macmillan K. The Edmonton Symptom Assessment System (ESAS): a simple method for the assessment of palliative care patients. Journal of Palliative Care 1991;7(2):6-9.

\section{Cancer Research UK 2015}

Cancer Research UK. Brain, other CNS and intracranial tumours incidence by anatomical site. Last reviewed: 17 December 2015. www.cancerresearchuk.org/health-professional/cancerstatistics/statistics-by-cancer-type/brain-other-cns-andintracranial-tumours/incidence\#ref-4 (accessed 12 December 2018).

\section{Catt 2008}

Catt S, Chalmers A, Fallowfield L. Psychosocial and supportive-care needs in high-grade glioma. Lancet. Oncology 2008;9(9):884-91. [PUBMED: 18760244]

\section{Cella 1993}

Cella DF, Tulsky DS, Gray G, Sarafian B, Linn E, Bonomi A, et al. The Functional Assessment of Cancer Therapy Scale: development and validation of the general measure. Journal of Clinical Oncology 1993;11(3):570-9.

\section{Cohen 1995}

Cohen SR, Mount BM, Strobel MG, Bui F. The McGill Quality of Life Questionnaire: a measure of quality of life appropriate for people with advanced disease. A preliminary study of validity and acceptability. Palliative Medicine 1995;9(3):207-19.

\section{Cohen 2006}

Cohen R, Leis AM, Kuhl D, Charbonneau C, Ritvo P, Ashbury FD. QOLLTI-F: measuring family carer quality of life. Palliative Medicine 2006;20(8):755-67.

\section{Collins 2014}

Collins A, Lethborg C, Brand C, Gold M, Moore G, Sundararajan V, et al. The challenges and suffering of caring for people with primary malignant glioma: qualitative perspectives on improving current supportive and palliative care practices. BMJ Supportive \& Palliative Care 2014;4(1):68-76. [PUBMED: 24644774]

\section{Davis 2015}

Davis MP, Temel JS, Balboni T, Glare P. A review of the trials which examine early integration of outpatient and home palliative care for patients with serious illnesses. Annals of Palliative Medicine 2015;4(3):99-121.

\section{Deeks 2011}

Deeks JJ, Higgins JPT, Altman DG. Chapter 9: Analysing data and undertaking meta-analyses. In: Higgins JP, Green S, editor(s). Cochrane Handbook for Systematic Reviews of Interventions Version 5.1.0 (updated March 2011). The Cochrane Collaboration, 2011. Available from handbook.cochrane.org. 5.1.0. The Cochrane Collaboration.

\section{Egger 1998}

Egger M, Smith GD. Bias in location and selection of studies. BMJ (Clinical Research Ed.) 1998;316(7124):61-6. [PUBMED: 9451274]

\section{Faithfull 2005}

Faithfull S, Cook K, Lucas C. Palliative care of patients with a primary malignant brain tumour: case review of service use and support provided. Palliative Medicine 2005;19(7):545-50. [PUBMED: 16295287]

\section{Ferlay 2015}

Ferlay J, Soerjomataram I, Dikshit R, Eser S, Mathers C, Rebelo $\mathrm{M}$, et al. Cancer incidence and mortality worldwide: Sources, methods and major patterns in GLOBOCAN 2012. International Journal of Cancer 2015;136(5):E386-59.

\section{Ferrell 2016}

Ferrell BR, Temel JS, Temin S, Smith TJ. Integration of palliative care into standard oncology care: ASCO clinical practice guideline update summary. Journal of Oncology Practice 2016;13(2):119-21.

\section{Ford 2012}

Ford E, Catt S, Chalmers A, Fallowfield L. Systematic review of supportive care needs in patients with primary malignant 
brain tumors. Neuro-Oncology 2012;14(4):392-404. [PUBMED: 22307475]

\section{Girgis 2011}

Girgis A, Lambert S, Lecathelinais C. The supportive care needs survey for partners and caregivers of cancer survivors: development and psychometric evaluation. Psycho-oncology 2011;20(4):387-93.

\section{Goodenberger 2012}

Goodenberger ML, Jenkins RB. Genetics of adult glioma. Cancer Genetics 2012;205(12):613-21.

\section{GRADEpro GDT 2014 [Computer program]}

McMaster University (developed by Evidence Prime). GRADEpro GDT. Version accessed prior to 05 Aug 2019. Hamilton (ON): McMaster University (developed by Evidence Prime), 2014.

\section{Groenvold 2017}

Groenvold M, Petersen MA, Damkier A, Neergaard MA, Nielsen JB, Pedersen L, et al. Randomised clinical trial of early specialist palliative care plus standard care versus standard care alone in patients with advanced cancer: The Danish Palliative Care Trial. Palliative Medicine 2017;31(9):814-24.

\section{Hamilton 1960}

Hamilton M. A rating scale for depression. Journal of Neurology, Neurosurgery, and Psychiatry 1960;23:56-62.

\section{Harding 2010}

Harding R, Higginson IJ, PRISMA. PRISMA: a pan-European co-ordinating action to advance the science in end-of-life cancer care. European Journal of Cancer 2010;46(9):1493-501. [PUBMED: 20185295]

\section{Haun 2017}

Haun MW, Estel S, Rücker G, Friederich HC, Villalobos M, Thomas M, et al. Early palliative care for adults with advanced cancer. Cochrane Database of Systematic Reviews 2017, Issue 6. [DOI: 10.1002/14651858.CD011129.pub2]

\section{Hearn 1999}

Hearn J, Higginson IJ. Development and validation of a core outcome measure for palliative care: the palliative care outcome scale. Palliative Care Core Audit Project Advisory Group. Quality in Health Care: QHC 1999;8(4):219-27.

\section{Higgins 2011}

Higgins JPT, Deeks JJ. Chapter 7: Selecting studies and collecting data. In: Higgins JP, Green S, editor(s). Cochrane Handbook for Systematic Reviews of Interventions Version 5.1.0 (updated March 2011). The Cochrane Collaboration, 2011. Available from handbook.cochrane.org. The Cochrane Collaboration.

\section{Higginson 2010}

Higginson IJ, Evans CJ. What is the evidence that palliative care teams improve outcomes for cancer patients and their families?. Cancer Journal (Sudbury, Mass.) 2010;16(5):423-35. [PUBMED: 20890138]

\section{Jacobs 2014}

Jacobs DI, Kumthekar P, Stell BV, Grimm SA, Rademaker AW, Rice L, et al. Concordance of patient and caregiver reports in evaluating quality of life in patients with malignant gliomas and an assessment of caregiver burden. Neuro-Oncology Practice 2014;1(2):47-54. [PUBMED: 26034616]

\section{Kavalieratos 2016}

Kavalieratos D, Corbelli J, Zhang D, Dionne-Odom JN, Ernecoff NC, Hanmer J, et al. Association between palliative care and patient and caregiver outcomes: a systematic review and meta-analysis. JAMA 2016;316(20):2104-14. [PUBMED: 27893131]

\section{Khan 2015}

Khan F, Amatya B, Ng L, Drummond K, Galea M. Multidisciplinary rehabilitation after primary brain tumour treatment. Cochrane Database of Systematic Reviews 2015, Issue 8. [DOI: 10.1002/14651858.CD009509.pub3]

\section{Kristjanson 1993}

Kristjanson LJ. Validity and reliability testing of the FAMCARE Scale: measuring family satisfaction with advanced cancer care. Social Science \& Medicine 1993;36(5):693-701.

\section{Kristjanson 2006}

Kristjanson LJ, Aoun SM, Oldham L. Palliative care and support for people with neurodegenerative conditions and their carers. International Journal of Palliative Nursing 2006;12(8):368-77. [PUBMED: 17077795]

\section{Langendam 2013}

Langendam MW, Akl EA, Dahm P, Glasziou P, Guyatt G, Schünemann HJ. Assessing and presenting summaries of evidence in Cochrane Reviews. Systematic Reviews 2013;2:81.

\section{Lin 2012}

Lin E, Rosenthal MA, Le BH, Eastman P. Neuro-oncology and palliative care: a challenging interface. Neuro-Oncology 2012;14(Suppl 4):iv3-7. [PUBMED: 23095828]

\section{Lipsman 2007}

Lipsman N, Skanda A, Kimmelman J, Bernstein M. The attitudes of brain cancer patients and their caregivers towards death and dying: a qualitative study. BMC Palliative Care 2007;6:7. [PUBMED: 17996072]

\section{Long 2016}

Long A, Halkett GKB, Lobb EA, Shaw T, Hovey E, Nowak AK. Carers of patients with high-grade glioma report high levels of distress, unmet needs, and psychological morbidity during patient chemoradiotherapy. Neuro-Oncology Practice 2016;3(2):105-12.

\section{Louis 2016}

Louis DN, Perry A, Reifenberger G, von Deimling A, FigarellaBranger D, Cavenee WK, et al. The 2016 World Health Organization Classification of Tumors of the Central Nervous System: a summary. Acta Neuropathologica 2016;131(6):803-20. [PUBMED: 27157931] 


\section{Lyons 2009}

Lyons KD, Bakitas M, Hegel MT, Hanscom B, Hull J, Ahles TA. Reliability and validity of the Functional Assessment of Chronic Illness Therapy-Palliative care (FACIT-Pal) scale. Journal of Pain Symptom Management 2009;37(1):23-32.

\section{MacDonald 2015}

MacDonald L, on behalf of the Neuro-Oncology Group. Top 10 priorities for clinical research in primary brain and spinal cord tumours. Final Report of the James Lind Alliance. June 2015. www.jla.nihr.ac.uk/priority-setting-partnerships/neurooncology/downloads/Neuro-Oncology-Group-Final-ReportJune-2015.pdf (accessed prior to 05 Aug 2019).

\section{McConigley 2012}

McConigley R, Aoun S, Kristjanson L, Colyer S, Deas K, O'Connor M, et al. Implementation and evaluation of an education program to guide palliative care for people with motor neurone disease. Palliative Medicine 2012;26(8):994-1000. [PUBMED: 22064045]

\section{Meader 2014}

Meader N, King K, Llewellyn A, Norman G, Brown J, Rodgers M, et al. A checklist designed to aid consistency and reproducibility of GRADE assessments: development and pilot validation. Systematic Reviews 2014;3:82.

\section{Moore 2013}

Moore G, Collins A, Brand C, Gold M, Lethborg C, Murphy M, et al. Palliative and supportive care needs of patients with highgrade glioma and their carers: a systematic review of qualitative literature. Patient Education and Counseling 2013;91(2):141-53. [PUBMED: 23218925]

\section{NCHSPCS 2002}

National Council for Hospice and Specialist Palliative Care Services (NCHSPCS). Briefing bulletin 11 eBook: Definitions of Supportive and Palliative Care. London: NCHSPCS, 2002.

\section{NICE 2006}

National Institute for Heath and Clinical Excellence (NICE). Guidance on Cancer Services. Improving outcomes for people with brain and other CNS tumours. The Manual. London: National Institute for Clinical Excellence, 2006:115-16.

\section{NICE 2018}

National Institute of Health and Care Excellence (NICE). Brain tumours (primary) and brain metastases in adults. 2018. https:// pathways.nice.org.uk/pathways/brain-cancers (accessed 4 July 2019).

\section{Osoba 1996}

Osoba D, Aaronson NK, Muller M, Sneeuw K, Hsu MA, Yung WK, et al. The development and psychometric validation of a brain cancer quality-of-life questionnaire for use in combination with general cancer-specific questionnaires. Quality of Life Research 1996;5(1):139-50.

\section{Philip 2015}

Philip J, Collins A, Brand CA, Gold M, Moore G, Sundararajan V, et al. Health care professionals' perspectives of living and dying with primary malignant glioma: implications for a unique cancer trajectory. Palliative \& Supportive Care 2015;13(6):1519-27. [PUBMED: 24138726]

\section{Radbruch 2009}

Radbruch L, Payne S. White paper on standards and norms for hospice and palliative care in Europe: part 1. European Journal of Palliative Care 2009;16(6):278-89.

\section{Reeves 2011}

Reeves BC, Deeks JJ, Higgins JPT, Wells GA. Chapter 13: Including non-randomized studies. In: Higgins JP, Green S, editor(s). Cochrane Handbook for Systematic Reviews of Interventions Version 5.1.0 (updated March 2011). The Cochrane Collaboration, 2011. Available from handbook.cochrane.org. The Cochrane Collaboration.

\section{Review Manager 2014 [Computer program]}

Nordic Cochrane Centre, The Cochrane Collaboration. Review Manager 5 (RevMan 5). Version 5.3. Copenhagen: Nordic Cochrane Centre, The Cochrane Collaboration, 2014.

\section{Rietjens 2017}

Rietjens JA, Sudore RL, Connolly M, van Delden JJ, Drickamer MA, Droger M, et al. Definition and recommendations for advance care planning: an international consensus supported by the European Association for Palliative Care. Lancet Oncology 2017;18(9):e543-51.

\section{Robinson 1983}

Robinson B. Validation of a Caregiver Strain Index. Journal of Gerontology 1983;38(3):344-8.

\section{Samarel 1996}

Samarel N, Leddy SK, Greco K, Cooley ME, Torres SC, Tulman L, et al. Development and testing of the symptom experience scale. Journal of Pain and Symptom Management 1996;12(4):221-8.

\section{Schünemann 2011}

Schünemann HJ, Oxman AD, Higgins JPT, Vist GE, Glasziou P, Guyatt GH. Chapter 11: Presenting results and 'Summary of findings' tables. In: Higgins JP, Green S, editor(s). Cochrane Handbook for Systematic Reviews of Interventions Version 5.1.0 (updated March 2011). The Cochrane Collaboration, 2011. Available from handbook.cochrane.org.

\section{Seng 2010}

Seng BK, Luo N, Ng WY, Lim J, Chionh HL, Goh J, et al. Validity and reliability of the Zarit Burden Interview in assessing caregiving burden. Annals of the Accademy of Medicine, Singapore 2010;39(10):758-63.

\section{Shuster 2011}

Shuster JJ. Review: Cochrane handbook for systematic reviews for interventions, Version 5.1.0, published 3/2011. Julian PT Higgins and Sally Green, Editors. Research Synthesis Methods 2011;2(2):126-30. 


\section{Steinhauser 2004}

Steinhauser KE, Clipp EC, Bosworth HB, Mcneilly M, Christakis NA, Voils $\mathrm{Cl}$, et al. Measuring quality of life at the end of life: validation of the QUAL-E. Palliative \& Supportive Care 2004;2(1):3-14.

\section{Sterne 2016}

Sterne JA, Hernán MA, Reeves BC, Savović J, Berkman ND, Viswanathan M, et al. ROBINS-I: a tool for assessing risk of bias in non-randomised studies of interventions. BMJ 2016;355:i4919.

\section{Stupp 2009}

Stupp R, Hegi ME, Mason WP, van den Bent MJ, Taphoorn MJ, Janzer RC, et al. Effects of radiotherapy with concomitant and adjuvant temozolomide versus radiotherapy alone on survival in glioblastoma in a randomised phase III study: 5-year analysis of the EORTC-NCIC trial. Lancet. Oncology 2009;10(5):459-66. [PUBMED: 19269895]

\section{Stupp 2010}

Stupp R, Tonn JC, Brada M, Pentheroudakis G. High-grade malignant glioma: ESMO Clinical Practice Guidelines for diagnosis, treatment and follow-up. Annals of Oncology 2010;21(Suppl 5):v190-3. [PUBMED: 20555079]

\section{Temel 2010}

Temel JS, Greer JA, Muzikansky A, Gallagher ER, Admane S, Jackson VA, et al. Early palliative care for patients with metastatic non-small-cell lung cancer. New England Journal of Medicine 2010;363(8):733-42. [PUBMED: 20818875]

\section{Temel 2017}

Temel JS, Greer JA, El-Jawahri A, Pirl WF, Park ER, Jackson VA, et al. Effects of early integrated palliative care in patients with lung and GI cancer: a randomized clinical trial. Journal of Clinical Oncology 2017;35(8):834-41. [PUBMED: 28029308]

\section{Tran 2010}

Tran B, Rosenthal MA. Survival comparison between glioblastoma multiforme and other incurable cancers. Journal of Clinical Neuroscience 2010;17(4):417-21. [PUBMED: 20167494]

\section{Vierhout 2017}

Vierhout M, Daniels M, Mazzotta P, Vlahos J, Mason WP, Bernstein M. The views of patients with brain cancer about palliative care: a qualitative study. Current Oncology 2017;24(6):374-82. [PUBMED: 29270049]

\section{Ware 1992}

Ware Jr JE, Sherbourne CD. The MOS 36-item short-form health survey (SF-36): I. Conceptual framework and item selection. Medical Care 1992;30(6):473-83.

\section{Wasner 2013}

Wasner M, Paal P, Borasio GD. Psychosocial care for the caregivers of primary malignant brain tumor patients. Journal of Social Work in End-of-Life \& Palliative Care 2013;9(1):74-95. [PUBMED: 23438646]

\section{Weitzner 1995}

Weitzner MA, Meyers CA, Gelke CK, Byrne KS, Cella DF, Levin VA. The Functional Assessment of Cancer Therapy (FACT) scale. Development of a brain subscale and revalidation of the general version (FACT-G) in patients with primary brain tumors. Cancer 1995;75(5):1151-61.

\section{WHO 2018}

World Health Organization (WHO). Palliative care. Fact sheet. 19 February 2018. www.who.int/news-room/fact-sheets/detail/ palliative-care (accessed 2 July 2019).

\section{Zigmond 1983}

Zigmond AS, Snaith RP. The hospital anxiety and depression scale. Acta Psychiatrica Scandinavica 1983;67(6):361-70.

\section{Zimmermann 2014}

Zimmermann C, Swami N, Krzyzanowska M, Hannon B, Leighl N, Oza A, et al. Early palliative care for patients with advanced cancer: a cluster-randomised controlled trial. Lancet 2014;383(9930):1721-30. [PUBMED: 24559581]

\section{AP P E N D I C E S}

Appendix 1. Draft 'Summary of findings' table for participants

\section{Title: Early palliative interventions for improving outcomes in people with a primary malignant brain tumour and their carers}

Participant or population: adults (18 years and older) with confirmed or historical diagnosis of a primary brain tumour, or both Settings: community and secondary care settings Intervention: early specialist or general palliative care Comparison: usual care and waiting list control 
(Continued)

\begin{tabular}{|c|c|c|c|c|c|c|}
\hline \multirow[t]{2}{*}{ Outcomes } & \multicolumn{2}{|c|}{$\begin{array}{l}\text { Illustrative compara- } \\
\text { tive risks* }\end{array}$} & \multirow{2}{*}{$\begin{array}{l}\text { Relative } \\
\text { effect } \\
(95 \% \mathrm{CI})\end{array}$} & \multirow{2}{*}{$\begin{array}{l}\text { No of par- } \\
\text { ticipants } \\
\text { (studies) }\end{array}$} & \multirow{2}{*}{$\begin{array}{l}\text { Quality of } \\
\text { evidence } \\
\text { (GRADE) }\end{array}$} & \multirow[t]{2}{*}{ Comment } \\
\hline & $\begin{array}{l}\text { Assumed } \\
\text { risk }\end{array}$ & $\begin{array}{l}\text { Corre- } \\
\text { sponding } \\
\text { risk }\end{array}$ & & & & \\
\hline
\end{tabular}

1. Survival from time of enrolment, to include one year and overall survival.

2. Quality of Life using validated Quality of Life tools;qualitative analysis of participant experience using validated and clearly described methodologies.

3. Symptom burden using validated symptom assessment tools; physical and cognitive function using validated assessment tools.

\section{Psychological outcomes including anxiety and depression using validated assessment tools.}

5. Care coordination and information giving based on qualitative assessment of participant feedback and/or objective measures of satisfaction.

6. Receipt of planned anticancer treatment: completion of initial neuro-oncology MDT treatment plan.

7. Resource use to include hospital and hospice utilisation measured in length of inpatient stay in days, number of outpatient appointments.

*The basis for the assumed risk (e.g. the median control group risk across studies) is provided in footnotes. The corresponding risk (and its $95 \% \mathrm{Cl}$ ) is based on the assumed risk in the comparison group and the relative effect of the intervention (and its $95 \% \mathrm{Cl}$ ). Abbreviations: $\mathrm{Cl}$ : confidence interval; HR: hazard ratio; MD: mean difference; RR: risk ratio; OR: odds ratio

\section{GRADE Working Group grades of evidence}

High-certainty: we are very confident that the true effect lies close to that of the estimate of the effect.

Moderate-certainty: we are moderately confident in the effect estimate. The true effect is likely to be close to the estimate of the effect, but there is a possibility that it is substantially different.

Low-certainty: our confidence in the effect estimate is limited. The true effect may be substantially different from the estimate of the effect.

Very low-certainty: we have very little confidence in the effect estimate. The true effect is likely to be substantially different from the estimate of effect

\section{Appendix 2. Draft 'Summary of findings' table for carers}

\section{Title: Early palliative interventions for improving outcomes in people with a primary malignant brain tumour and their carers}


(Continued)

Participant or population: informal adult carers of people with a confirmed diagnosis of a primary brain tumour: individual and family level

Settings: community and secondary care settings

Intervention: early specialist or general palliative care

Comparison: usual care and waiting list control

\begin{tabular}{lllll}
\hline Outcomes & $\begin{array}{l}\text { Illustrative compara- } \\
\text { tive risks* }\end{array}$ & $\begin{array}{l}\text { Relative } \\
\text { effect } \\
(95 \% \mathrm{Cl})\end{array}$ & $\begin{array}{l}\text { No of partic- } \\
\text { ipants (stud- } \\
\text { ies) }\end{array}$ & $\begin{array}{l}\text { Quality of } \\
\text { evidence } \\
\text { (GRADE) }\end{array}$ \\
\cline { 2 - 4 } & $\begin{array}{l}\text { Assumed } \\
\text { risk }\end{array}$ & $\begin{array}{l}\text { Corre- } \\
\text { sponding } \\
\text { risk }\end{array}$ & \\
\hline
\end{tabular}

\footnotetext{
1. Psychological outcomes including anxi-

ety and depression using validated assessment tools
}

2. Care coordination and information giving based on qualitative assessment of carer feedback or objective measures of satisfaction, or both

\section{Qualitative analysis of carer experi- ence using clearly described and validated methodologies}

\section{Bereavement outcomes using validated measures}

\section{Resource use and costs including oppor- tune costs of loss of income}

*The basis for the assumed risk (e.g. the median control group risk across studies) is provided in footnotes. The corresponding risk (and its $95 \% \mathrm{Cl}$ ) is based on the assumed risk in the comparison group and the relative effect of the intervention (and its $95 \% \mathrm{Cl}$ ). Abbreviations: $\mathrm{Cl}$ : confidence interval; HR: hazard ratio; MD: mean difference; RR: risk ratio; OR: odds ratio.

\section{GRADE Working Group grades of evidence}

High-certainty: we are very confident that the true effect lies close to that of the estimate of the effect.

Moderate-certainty: we are moderately confident in the effect estimate. The true effect is likely to be close to the estimate of the effect, but there is a possibility that it is substantially different.

Low-certainty: our confidence in the effect estimate is limited. The true effect may be substantially different from the estimate of the effect.

Very low-certainty: we have very little confidence in the effect estimate. The true effect is likely to be substantially different from the estimate of effect.

\section{Appendix 3. MEDLINE search strategy}

\section{Ovid MEDLINE}

1. exp Palliative Care/

2. exp Terminal Care/

3. exp Terminally III/

Early palliative interventions for improving outcomes in people with a primary malignant brain tumour and their carers (Protocol) 
4. exp Hospices/

5. exp Hospice Care/

6. exp "Hospice and Palliative Care Nursing"/

7. ("last year of life" or LYOL or "life's end").ti,ab.

8. ((macmillan or marie curie or district) adj nurs $\left.{ }^{\star}\right) . \mathrm{mp}$.

9. (hospice ${ }^{\star}$ or (nursing adj3 home*)).mp.

10. exp Palliative Medicine/

11. exp Advance Care Planning/

12. $\left(\right.$ advance $^{\star}$ adj5 care plan $\left.{ }^{\star}\right) . t i, a b$.

13. (future adj5 care plan ${ }^{\star}$ ).ti,ab.

14. exp Caregivers/

15. ((early or specialist or general or primary) adj5 palliat $\left.{ }^{\star}\right)$.ti,ab.

16. (support ${ }^{\star}$ adj3 care).ti,ab.

17. palliat ${ }^{\star}$. tw.

18. "advanced disease ${ }^{\star}$.tw.

19. ("end-stage disease ${ }^{\star "}$ or "end stage disease* or end-stage illness" or "end stage").tw.

20. (end adj6 life).tw.

21. (terminal ${ }^{\star}$ adj6 (disease* or ill $^{\star}$ or care $\left.{ }^{\star}\right)$ ).ti,ab.

22. ("terminal-stage ${ }^{\star}$ or dying).ti,ab.

23. (close adj6 death).tw.

24. 1 or 2 or 3 or 4 or 5 or 6 or 7 or 8 or 9 or 10 or 11 or 12 or 13 or 14 or 15 or 16 or 17 or 18 or 19 or 20 or 21 or 22 or 23

25. ((home* or in-home* or domicile or outreach or residential or housing or posthospital or post-hospital or communit* or moblie or ambulatory or door to door) adj2 (team ${ }^{\star}$ or centre or center $^{\star}$ or treat ${ }^{\star}$ or care or interven* or therap* or manag* or model* or program ${ }^{\star}$

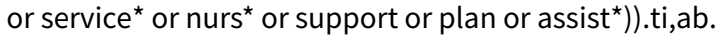

26. (self adj3 (help* or care)).ti,ab.

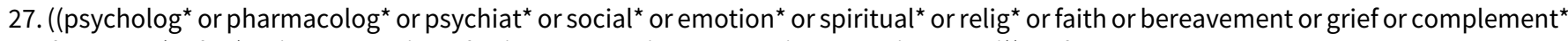
or alternative) adj3 (car ${ }^{\star}$ or service ${ }^{\star}$ or plan ${ }^{\star}$ or interven ${ }^{\star}$ or support ${ }^{\star}$ or assist ${ }^{\star}$ or $\left.\left._{\text {prog }}^{\star}\right)\right)$.ti,ab.

28. (symptom ${ }^{\star}$ adj3 (care or manag* or support $^{\star}$ or modif* or control $^{\star}$ or assess $\left.{ }^{\star}\right)$ ).ti,ab.

29. 25 or 26 or 27 or 28

30. exp Brain Neoplasms/

31. exp Glioma/

32. (brain adj3 (tumor ${ }^{\star}$ or tumour ${ }^{\star}$ or neoplas* or malignan $^{\star}$ or cancer $^{\star}$ or carcinoma $\left.^{\star}\right)$ ).ti,ab.

33. (glioma* or astrocytoma* or meningioma* or oligodendroglioma* or oligoastrocytoma* or glioblastoma* or GBM* or Glioblastoma multiforme or "Primary brain tumo? $\left.r^{\star} "\right) . t i, a b$.

34.30 or 31 or 32 or 33

35. (early or advance* or forward or simultaneous).ti,ab.

36. 24 or 29

37.35 and 36

38. 34 and 37

\section{CONTRIBUTIONS OF AUTHORS}

$\mathrm{AB}$ drafted the protocol.

SS drafted the protocol.

$\mathrm{HF}$ completed protocol updates and modifications.

ATB completed protocol updates and modifications.

FM contributed to drafting the protocol.

AN contributed to drafting the protocol.

$M B$ contributed to drafting the protocol.

$\mathrm{HB}$ contributed to drafting the protocol.

All authors agreed on the final version of the protocol for publication.

\section{DECLARATIONS OF INTEREST}

Anthony Byrne has no known conflicts of interest. Stephanie Sivell has no known conflicts of interest. Fabio Ynoe Moraes has no known conflicts of interest. Helen Bulbeck has no known conflicts of interest. Anna Torrens-Burton has no known conflicts of interest. Mark Bernstein has no known conflicts of interest. Annmarie Nelson has no known conflicts of interest. Helen Fielding has no known conflicts of interest. 


\section{SOURCES OF SUPPORT}

\section{Internal sources}

- Marie Curie, UK.

AB, AN, SS posts are supported by Marie Curie Cancer Care core grant funding (grant reference: MCCC-FCO-11-C).

\section{External sources}

- None, Other. 